\title{
A topological-like model for gravity in 4D space-time
}

\author{
Ivan Morales ${ }^{\mathrm{a}}$, Bruno Neves ${ }^{\mathrm{b}}$, Zui Oporto $^{\mathrm{c}}$, Olivier Piguet ${ }^{\mathrm{d}}$ \\ Departamento de Física, Universidade Federal de Viçosa-UFV, Viçosa, MG, Brazil
}

Received: 3 March 2016 / Accepted: 21 March 2016 / Published online: 9 April 2016

(C) The Author(s) 2016. This article is published with open access at Springerlink.com

\begin{abstract}
In this paper we consider a model for gravity in four-dimensional space-time originally proposed by Chamseddine, which may be derived by dimensional reduction and truncation from a five-dimensional Chern-Simons theory. Its topological origin makes it an interesting candidate for an easier quantization, e.g., in the loop quantization framework. The present paper is dedicated to a classical analysis of the model's properties. Cosmological solutions as well as wave solutions are found and compared with the corresponding solutions of Einstein's general relativity with cosmological constant.
\end{abstract}

\section{Introduction}

Gravitation as described by Einstein's general relativity is notoriously difficult to reconcile with quantum theory, a task which is nevertheless necessary if one want to understand physics at the very small scale defined by the Planck length $l_{\mathrm{P}} \sim 10^{-35} \mathrm{~m}$, also in the hope that quantum mechanics will cure the singularities of the classical theory such as the Big Bang and Black Hole ones. Loop quantum gravity [1-3] (LQG) is one attempt to do it. It starts from Einstein's classical general relativity (GR) in the Ashtekar-Barbero formalism where the dynamical variables are an SU(2) YangMills type connection together with its canonical conjugate momentum field. The dynamics is expressed as a set of constraints which correspond to the gauge invariances of the theory [4,5], namely $\mathrm{SU}(2)$ local invariance and the invariances under the space and time diffeomorphisms. The quantum theory is then defined by constructing a Hilbert space whose elements are certain wave functionals of the connection obeying the constraints. The latter should be well defined as self-

\footnotetext{
a e-mail: mblivan@gmail.com

be-mail: bruno.lqg@gmail.com

c e-mail: azurnasirpal@ gmail.com

de-mail: opiguet@pq.cnpq.br
}

adjoint operators, and then solved in the sense that they select the physical wave functionals as those which are annihilated by them. The main difficulty is in the definition of the time diffeomorphism constraint and its solution. Much progress has been made by Thiemann et al. [2], and more recently by Rovelli et al. in the spin foam formalism [3], leading to very promising results.

General relativity is a "background invariant theory", which means that no a priori geometric structure is given to the space-time manifold where the theory is defined: the metric belongs to the dynamical fields. Another class of background-independent theories is provided by topological theories such as the Chern-Simons (CS) theories. ${ }^{1}$ Remarkably enough [6], gravity in three space-time dimensions can be written as a CS theory whose gauge group is the local Poincaré group ISO $(1,2)$, but also $\mathrm{SO}(1,3)$ or $\mathrm{SO}(2,2)$ if there is a positive or negative cosmological constant. The question is: Could one describe higher-dimension gravity as a CS theory [7-14]? An essential difference between gravity in 3D and gravities in more than three dimensions is that the former has no local degree of freedom, whereas the latter do. The same happens for the CS theories in 3D and in more than three dimensions. Since CS theories live in odd-dimensional space-times, the first one which admits local degrees of freedom is the one in $5 \mathrm{D}$, with the gauge group $\operatorname{ISO}(1,4), \operatorname{SO}(1,5)$ or $\mathrm{SO}(2,4)$ (Poincaré, de Sitter or anti-de Sitter).

An advantage of topological theories-with the gauge groups mentioned above-is that (some of) the diffeomorphism invariances are consequences of the gauge invariances. For $\mathrm{CS}$ in $3 \mathrm{D}$, all diffeomorphism invariances follow on shell from gauge invariance $[15,16]$, whereas, in $5 \mathrm{D}$, only the invariance under the time diffeomorphisms follows on shell $[17,18]$. In the latter case this means that the constraint associated with the time diffeomorphisms is a consequence

\footnotetext{
${ }^{1}$ We could also mention the BF theories. However, unless some constraints are applied to them, they have to address local degrees of freedom in any space-time dimension.
} 
of the other constraints. Thus the difficult task of defining and solving the diffeomorphism constraint in the 5D quantum gravity described by this CS theory would be avoided.

Being interested in 4D gravity, can we find a similar, topological-like theory? An answer has been provided by Chamseddine [13]: a theory which, beyond containing the gravitation fields is also containing a dilaton-type scalar field. It can be derived from the 5D CS theory by dimensional reduction and truncation of some of the component fields. As we will show, the set of solutions of the Chamseddine model is a subset of the solutions of the complete, non-truncated 5D CS theory reduced to 4D. As such, in view of the interesting properties concerning the constraints mentioned above, it is worthwhile to study the classical aspects of the Chamseddine model, which is the purpose of the present work. A study of the whole 5D CS theory, with or without Kaluza-Klein dimensional reduction, classically and quantum mechanically, will be reported elsewhere [19]. More specifically, the proposal of the present work is to investigate the dynamics of the Chamseddine model and compare some of its solutions with solutions of the conventional Einstein theory. We will in particular focus on solutions of the cosmological type and wave solutions.

The present paper begins in Sect. 2 with a review of Chamseddine's derivation of his 4D model-whose gauge invariance is de Sitter $\mathrm{SO}(1,4)$ or anti-de Sitter $\mathrm{SO}(2,3)$ from a $\mathrm{SO}(1,5)$ or $\mathrm{SO}(2,4) 5 \mathrm{D}$ Chern-Simons theory by dimensional reduction and truncation of some fields. We clarify some points of this truncation, and, moreover, show through a well-chosen gauge fixing that the model is a theory of a dilaton-like scalar field interacting with a gravitational field with torsion. We also show here that the field equations of the Chamseddine theory are special solutions of the full untruncated CS theory reduced to 4D. Linear approximations are studied in Sect. 3, leading to the Newtonian limit and to gravitational wave solutions. Section 4 is devoted to the study of cosmological solutions of the theory and their comparison with the $\Lambda \mathrm{CDM}$ model. Conclusions and outlooks are presented in Sect. 5. Conventions and notations are displayed in an appendix.

\section{The Chamseddine model}

\subsection{D (A)dS Chern-Simons theory as a theory of gravity in $5 \mathrm{D}$}

We start with a description of the five-dimensional ChernSimons (CS) theory for the (anti-)de Sitter gauge group and its interpretation as a gravitation theory [9-11] (see [12] for a comprehensive review). Our notations and conventions are summarized in the appendix.
The gauge group transformations are those which leave invariant the metric $\eta_{M N}=\operatorname{diag}(-1,1,1,1,1, s)$, with $M, N, \ldots=0, \ldots, 5$ and where $s$ takes the values \pm 1 . The signatures $(-1,1,1,1,1,1)$ and $(-1,1,1,1,1,-1)$ correspond, respectively, to the Minkowskian de Sitter group $\mathrm{SO}(1,5)$ and anti-de Sitter group $\mathrm{SO}(2,4)$ for $5 \mathrm{D}$ space-time. They will be collectively denoted ${ }^{2}$ by $(\mathrm{A}) \mathrm{dS}_{6}$.

A basis of the Lie algebra (a)ds 6 of $(\mathrm{A}) \mathrm{dS}_{6}$ is given by the generators $M_{M N}=-M_{N M}$, realized as the $6 \times 6$ matrices $\left(M_{M N}\right)^{P} Q:=-\delta_{M}^{P} \eta_{N Q}+\delta_{N}^{P} \eta_{M Q}$, obeying the commutation relations

$$
\begin{aligned}
{\left[M_{M N}, M_{P Q}\right]=} & \eta_{M P} M_{N Q}-\eta_{M Q} M_{N P}-\eta_{N P} M_{M Q} \\
& +\eta_{N Q} M_{M P} .
\end{aligned}
$$

The field variables ${ }^{3}$ of the theory are the components of a connection form $\hat{A}=\hat{A}_{\alpha} \mathrm{d} x^{\alpha}$, with values in the Lie algebra (a)ds. In the basis $\left(M_{M N}\right)$, the connection form reads

$\hat{A}=\frac{1}{2} \hat{A}^{M N} M_{M N}=\frac{1}{2} \hat{A}_{\alpha}^{M N} \mathrm{~d} x^{\alpha} M_{M N}$,

and it transforms as

$\delta \hat{A}^{M N}=\hat{d} \hat{\epsilon}^{M N}+\hat{A}^{M}{ }_{P} \hat{\epsilon}^{P N}-\hat{A}^{N}{ }_{P} \hat{\epsilon}^{P M}$

under the infinitesimal (A) $\mathrm{dS}_{6}$ gauge transformations. The gauge invariant $\mathrm{CS}$ action is given by ${ }^{4}$

$$
\begin{aligned}
& S_{\mathrm{CS}}=\frac{1}{24} \varepsilon_{M N P Q R S} \\
& \times \int_{\mathcal{M}_{5}}\left(\hat{A}^{M N} \hat{d} \hat{A}^{P Q_{\mathrm{d}}} \hat{A}^{R S}+\frac{3}{2} \hat{A}^{M N}\left(\hat{A}^{2}\right)^{P Q} \mathrm{~d} \hat{A}^{R S}\right. \\
& \left.+\frac{3}{5} \hat{A}^{M N}\left(\hat{A}^{2}\right)^{P Q}\left(\hat{A}^{2}\right)^{R S}\right) \text {. }
\end{aligned}
$$

$\varepsilon_{M N P Q R S}$ is the Levi-Civita totally antisymmetric tensor with the normalization condition ${ }^{5} \varepsilon_{012345}=1$. The field equations obtained by varying the connection $\hat{A}$ are

$\frac{1}{4} \varepsilon_{M N P Q R S} \hat{F}^{P Q} \hat{F}^{R S}=0$, where $\hat{F}^{P Q}=\hat{d} \hat{A}^{P Q}+\left(\hat{A}^{2}\right)^{P Q}$,

with $\hat{F}^{M N}=\hat{d} \hat{A}^{M N}+\hat{A}^{N}{ }_{P} \hat{A}^{P N}$ the Yang-Mills curvature.

In the same way as one can interpret the three-dimensional CS theory for the pseudo-orthogonal gauge group $\mathrm{SO}(1,3)$ or $\mathrm{SO}(2,2)$ as a gravitation theory with cosmological constant [6], one can indeed do the same here making the following identifications of the generators $M_{M N}$ with the five-

\footnotetext{
$\overline{2}$ The suffix $N$ in (A) $\mathrm{dS}_{N}$ makes explicit the dimension of the defining representation space.

${ }^{3}$ Fields and forms in five-dimensional space-time $\mathcal{M}_{5}$ are written with a hat, space-time indices being denoted by $\alpha, \beta, \ldots=0, \ldots, 4$.

${ }^{4}$ We do not write explicitly the wedge symbol $\wedge$ for the external products of forms. E.g., the product $\left(\hat{A}^{2}\right)^{P Q}=-\left(\hat{A}^{2}\right)^{Q P}$ stems from $\hat{A}^{P T} \wedge \hat{A}_{T} Q$.

5 Indices are lowered and raised using the metric $\eta_{M N}$.
} 
dimensional Lorentz generators $M_{A B}$ and "translation" generators $P_{A}$, with $A, B, \ldots=0, \ldots, 4$ :

$M_{A B}=M_{A B}, \quad P_{A}:=\lambda M_{A 5}$,

where $\lambda>0$ is a parameter with the dimension of a mass or of the inverse of a length in the system of units where $c=\hbar=1$. The commutation relations (2.1) take the explicit form of the (a) $\mathrm{ds}_{6}$ commutation relations (A.4), with $\eta_{A B}$ being the fivedimensional Lorentz metric $(-1,1,1,1,1)$ and $s\left|\lambda^{2}\right|$ playing the role of the "cosmological constant" (see Appendix A.2). We define accordingly the spin connection form $\hat{\omega}^{A B}$ and the 5-bein form $\hat{e}^{A}$ as

$\hat{\omega}^{A B}:=\hat{A}^{A B}, \quad \hat{e}^{A}:=\frac{1}{\lambda} \hat{A}^{A 5}$.

We can thus write the infinitesimal (A) $\mathrm{dS}_{6}$ gauge transformations (2.3) and the CS action (2.4) as ${ }^{6}$

$$
\begin{aligned}
\delta \hat{\omega}^{A B}= & \hat{d} \hat{\epsilon}^{A B}+\hat{\omega}^{A}{ }_{C} \hat{\epsilon}^{C B}-\hat{\omega}^{B}{ }_{C} \hat{\epsilon}^{C A} \\
& +\lambda\left(\hat{\epsilon}^{A} \hat{e}^{B}-\hat{\epsilon}^{B}{ }_{5}^{A}\right), \\
\delta \hat{e}^{A}= & \hat{e}^{C} \hat{\epsilon}_{C}^{A}+\frac{1}{\lambda}\left(\hat{d} \hat{\epsilon}^{A 5}+\hat{\omega}^{A} \hat{\epsilon}^{C 5}\right), \\
S^{(5 D)}= & \frac{\lambda}{8} \int_{\mathcal{M}_{5}} \varepsilon_{A B C D E} \\
& \times\left(\hat{e}^{A} \hat{R}_{(5)}^{B C} \hat{R}_{(5)}^{D E}-\frac{2 s \lambda^{2}}{3} \hat{e}^{A} \hat{e}^{B} \hat{e}^{C} \hat{R}_{(5)}^{D E}+\frac{\lambda^{4}}{5} \hat{e}^{A} \hat{e}^{B} \hat{e}^{C} \hat{e}^{D} \hat{e}^{E}\right) .
\end{aligned}
$$

We recognize in the second and third terms the standard Einstein-Hilbert and cosmological terms of 5D gravity, respectively. The novelty of the Chern-Simons action is the appearance of the first term, which is of the form $e \hat{R}_{(5)} \hat{R}_{(5)}$; this term does not enter through an arbitrary coupling constant, but instead through a rational number which is prefixed by the requirement of the theory to be $(A) \mathrm{dS}_{6}$ invariant, although the action is written in a manifestly Lorentz $\operatorname{SO}(1,4)$ invariant form.

Since later on we will proceed to a dimensional reduction from 5D to 4D, we will need the decomposition of the (A) $\mathrm{dS}_{6}$ group in terms of $4 \mathrm{D}$ Lorentz $\mathrm{SO}(1,3)$ representations, as displayed in (A.6, A.7). The connection components are accordingly decomposed as

${ }^{6}$ A subscript will be used to distinguish the (A) $\mathrm{dS}_{5}$ curvature from the Lorentzian $\mathrm{SO}(1,3)$ curvature. We opt not to write the subscript in the latter case. Thus we have

$\hat{R}_{(5)}^{A B}=\hat{d} \hat{\omega}^{A B}+\hat{\omega}_{C}^{A} \hat{\omega}^{C B}$,

$\hat{R}^{I J}=\hat{d} \hat{\omega}^{I J}+\hat{\omega}^{I}{ }_{K} \hat{\omega}^{K J}$.

Let us note that the latter is not just obtained by restricting the former to the indices $I, J$, instead we have

$\hat{R}_{(5)}^{I J}=\hat{R}^{I J}-\hat{\omega}^{4 I} \hat{\omega}^{4 J}$.
$\hat{\omega}^{A B}=\left\{\hat{\omega}^{I J}, \hat{\omega}^{I 4}=: \lambda \hat{b}^{I}\right\}, \quad \hat{e}^{A}=\left\{\hat{e}^{I}, \hat{e}^{4}\right\}$.

With these definitions, the action (2.9) is written

$$
\begin{aligned}
S^{(5 D)}= & \frac{1 \lambda}{8} \int_{\mathcal{M}_{5}} \varepsilon_{I J K L}\left(\hat{e}^{4}\left(\hat{R}^{I J}-\lambda^{2}\left(\hat{b}^{I} \hat{b}^{J}+s \hat{e}^{I} \hat{e}^{J}\right)\right)\right. \\
& \times\left(\hat{R}^{K L}-\lambda^{2}\left(\hat{b}^{K} \hat{b}^{L}+s \hat{e}^{K} \hat{e}^{L}\right)\right) \\
& +2 \lambda \hat{D} \hat{e}^{I} \hat{b}^{J}\left(\hat{R}^{K L}-\frac{2 \lambda^{2}}{3} \hat{b}^{K} \hat{b}^{L}\right) \\
& \left.-2 \lambda \hat{D} \hat{b}^{I} \hat{e}^{J}\left(\hat{R}^{K L}-\frac{2 s \lambda^{2}}{3} \hat{e}^{K} \hat{e}^{L}\right)\right)
\end{aligned}
$$

with $\hat{D}$ the covariant external derivative: $\hat{D} \hat{e}^{I}=\hat{d} \hat{e}^{I}+$ $\hat{\omega}^{I}{ }_{J} \hat{e}^{J}$, etc. , and $\hat{R}^{I J}=d \hat{\omega}^{I J}+\hat{\omega}^{I}{ }_{K} \hat{\omega}^{K J}$. Let us note that the fields $\hat{e}^{I}$ and $\hat{b}^{I}$ play a symmetrical role in the action, so in principle we can use any of the two to define a four-dimensional soldering form. A qualitative difference between these quantities will show up after a suitable truncation.

In view of the announced dimensional reduction, we make explicit a split of the $\mathrm{D}=5$ space-time coordinates in $\mathrm{D}=4$ space-time coordinates $x^{\mu}, \mu=0, \ldots, 3$ and the fifth coordinate $\chi:=x^{4}$ by writing the form fields as

$$
\begin{aligned}
\hat{e}^{I} & =e_{\mu}^{I} \mathrm{~d} x^{\mu}+e_{\chi}^{I} \mathrm{~d} \chi, \\
\hat{b}^{I} & =b_{\mu}^{I} \mathrm{~d} x^{\mu}+b_{\chi}^{I} \mathrm{~d} \chi, \\
\hat{e}^{4} & =e_{\mu}^{4} \mathrm{~d} x^{\mu}+e_{\chi}^{4} \mathrm{~d} \chi, \\
\hat{\omega}^{I J} & =\omega_{\mu}^{I J} \mathrm{~d} x^{\mu}+\omega_{\chi}^{I J} \mathrm{~d} \chi .
\end{aligned}
$$

The corresponding splits for the curvature components read $\hat{F}^{M N}=F^{M N}+F_{\chi}^{M N} \mathrm{~d} \chi$

where

$F^{M N}=\frac{1}{2} F_{\mu \nu}^{M N} \mathrm{~d} x^{\mu} \mathrm{d} x^{\nu}, \quad F_{\chi}^{M N}=F_{\mu \chi}^{M N} \mathrm{~d} x^{\mu}$.

We also have to split each of these forms in terms of their Lorentz $\mathrm{SO}(1,3)$ components,

$F^{M N}=\left(F^{I J}, F^{4 I}, F^{5 I}, F^{45}\right)$,

$F_{\chi}^{M N}=\left(F_{\chi}^{I J}, F_{\chi}^{4 I}, F_{\chi}^{5 I}, F_{\chi}^{45}\right)$.

Then with the relabeling (2.11), (2.12), the curvature components take the form

$$
\begin{aligned}
& F^{I J}=R^{I J}-\lambda^{2} b^{I} b^{J}-s \lambda^{2} e^{I} e^{J}, \\
& F^{I 4}=\lambda D b^{I}-s \lambda^{2} e^{I} e^{4}, \\
& F^{I 5}=\lambda D e^{I}+\lambda^{2} b^{I} e^{4}, \\
& F^{45}=\lambda d e^{4}-\lambda^{2} b_{I} e^{I}, \\
& F_{\chi}^{I J}=R_{\chi}^{I J}+\lambda^{2}\left(b_{\chi}^{I} b^{J}-b^{I} b_{\chi}^{J}\right)+s \lambda^{2}\left(e_{\chi}^{I} e^{J}-e^{I} e_{\chi}^{J}\right), \\
& F_{\chi}^{I 4}=\lambda\left(D b_{\chi}^{I}+\omega_{\chi}^{I} b^{J}\right)+s \lambda^{2}\left(e_{\chi}^{I} e^{4}-e^{I} e_{\chi}^{4}\right) \\
& F_{\chi}^{I 5}=\lambda\left(D e_{\chi}^{I}+\omega_{\chi}^{I} e^{J}\right)-\lambda^{2}\left(b_{\chi}^{I} e^{4}-b^{I} e_{\chi}^{4}\right), \\
& F_{\chi}^{45}=\lambda d e_{\chi}^{4}+\lambda^{2}\left(b_{\chi}^{I} e^{I}-b^{I} e_{\chi}^{I}\right),
\end{aligned}
$$


where $D$, and $R^{I J}$ represent the covariant exterior derivative and the curvature 2-form associated to the Lorentz connection $\omega^{I J}$. The field equations (2.5) are then split into 4-forms equations and 3 -form equations (the $\chi$ components). The 4form equations are

$\begin{array}{ll}\varepsilon_{I J K L}\left(F^{45} F^{K L}-2 F^{K 4} F^{L 5}\right) & =0, \\ \varepsilon_{I J K L} F^{J 5} F^{K L} & =0, \\ \varepsilon_{I J K L} F^{J 4} F^{K L} & =0, \\ \varepsilon_{I J K L} F^{I J} F^{K L} & =0,\end{array}$

and the 3 -form equations are

$$
\begin{aligned}
& \varepsilon_{I J K L}\left(F_{\chi}^{45} F^{K L}+F^{45} F_{\chi}^{K L}\right. \\
& \left.\quad-2 F_{\chi}^{K 4} F^{L 5}-2 F^{K 4} F_{\chi}^{L 5}\right)=0, \\
& \varepsilon_{I J K L}\left(F_{\chi}^{J 5} F^{K L}+F^{J 5} F_{\chi}^{K L}\right)=0, \\
& \varepsilon_{I J K L}\left(F_{\chi}^{J 4} F^{K L}+F^{J 4} F_{\chi}^{K L}\right)=0, \\
& \varepsilon_{I J K L} F^{I J} F_{\chi}^{K L}=0,
\end{aligned}
$$

with the curvature components given by (2.13).

This theory is still invariant under the full (A) $\mathrm{dS}_{6}$ transformations, which now read, in terms of the $5 \mathrm{D}$ "hat" quantities:

$\delta \hat{\omega}^{I J}=\hat{D} \hat{\epsilon}^{I J}+\lambda s\left(\hat{\epsilon}^{I 5} \hat{e}^{J}-\hat{\epsilon}^{J 5} \hat{e}^{I}\right)+\lambda\left(\hat{\epsilon}^{I 4} \hat{b}^{J}-\hat{\epsilon}^{J 4} \hat{b}^{I}\right)$,

$\delta \hat{e}^{I}=(1 / \lambda) \hat{D} \hat{\epsilon}^{I 5}+\hat{e}^{J} \hat{\epsilon}_{J}^{I}+\hat{b}^{I} \hat{\epsilon}^{45}-\hat{e}_{\chi}^{4} \hat{\epsilon}^{I 4}$,

$\delta \hat{b}^{I}=(1 / \lambda) \hat{D} \hat{\epsilon}^{I 4}+\hat{b}^{J} \hat{\epsilon}_{J}^{I}-s \hat{e}^{I} \hat{\epsilon}^{45}+s \hat{e}_{\chi}^{4} \hat{\epsilon}^{I 5}$,

$\delta \hat{e}^{4}=(1 / \lambda) \hat{d} \hat{\epsilon}^{45}-\hat{b}_{I} \hat{\epsilon}^{I 5}+\hat{e}_{I} \hat{\epsilon}^{I 4}$.

\section{A partial gauge fixing}

The action (2.9) or (2.10) and the field equations (2.14) and (2.15) may be simplified by a partial gauge fixing consisting of the eight conditions

$b_{\chi}^{I}=0, \quad e_{\chi}^{I}=0, \quad I=0, \ldots, 3$,

which fix the gauge symmetries generated by $M_{I 5}=P_{I} / \lambda$ and $M_{I 4}=Q_{I} / \lambda$, respectively, as can be inferred from the transformation laws (2.16) for the $\chi$ components of $\hat{e}^{I}$ and $\hat{b}^{I}$, the field $\hat{e}_{\chi}^{4}$ being assumed not to vanish. This reduces the explicit gauge symmetry to the group $\mathrm{SO}(1,3) \times(\mathrm{A}) \mathrm{dS}_{2}$, where $\mathrm{SO}(1,3)$ is the $4 \mathrm{D}$ Lorentz group and $(\mathrm{A}) \mathrm{dS}_{2}=\mathrm{U}(1)$ if $s>0$ (theory with positive cosmological constant) or the dilatation group if $s<0$ (theory with negative cosmological constant). Of course (2.17) is only a gauge fixing: the theory remains a full $(\mathrm{A}) \mathrm{dS}_{6}$ gauge theory.

\subsection{The Chamseddine action}

A 4D theory may be obtained trough a Kaluza-Klein dimensional compactification in which "matter-like" fields are realized as fifth-dimension components of the 5D fields. In our context we may assume that the fifth dimension, of the coordinate $x^{4}=\chi$, is compact and "microscopic", and the fields are expanded in Kaluza-Klein modes. In the present paper, we restrict the study to the zero-mode sector-which amounts to considering all fields as constant in $\chi$-leaving a complete discussion involving all modes for future work [19]. This means

$\partial_{\chi} f(x)=0, \quad \forall$ field $f$.

The Chamseddine model has been obtained [13] by a truncation consisting in setting some fields to zero:

$e_{\chi}^{I}=0, \quad \omega_{\chi}^{I J}=0, \quad e_{\mu}^{4}=0, \quad b_{\mu}^{I}=0$.

We may observe that the first condition is in fact nothing but a gauge fixing condition, the second of (2.17). The other three truncation conditions do indeed break $(\mathrm{A}) \mathrm{dS}_{6}$, apparently to $\mathrm{SO}(1,3)$. However, a reordering of the remaining fields in new multiplets allows one to show that the resulting theory actually has a hidden (A) $\mathrm{dS}_{5}$ gauge invariance [13]. In order to see this, one does not apply for the moment the first of the gauge fixing conditions (2.17), and one reorders the fields in (A) $\mathrm{dS}_{5}$ multiplets as

$\mathbb{A}^{A B}=\left\{\mathbb{A}^{I J}, \mathbb{A}^{I 4}\right\}:=\left\{\omega^{I J}, \lambda e^{I}\right\}$,
$\Phi^{A}=\left\{\Phi^{I}, \Phi^{4}\right\}:=\left\{-b_{\chi}^{I}, e_{\chi}^{4}\right\}$.

Using these definitions together with the truncation conditions (2.19), the action (2.9) or (2.10) reduces to the obviously $(\mathrm{A}) \mathrm{dS}_{5}$ invariant expression ${ }^{7}$

$S^{(4 D)}=\frac{1}{8} \int_{\mathcal{M}_{4}} \varepsilon_{A B C D E} \Phi^{A} \mathbb{F}^{B C} \mathbb{F}^{D E}$,

with the $(\mathrm{A}) \mathrm{dS}_{5}$ curvature

$\mathbb{F}^{A B}=d \mathbb{A}^{A B}+\mathbb{A}_{C}^{A} \mathbb{A}^{C B}$,

which in terms of the $\mathrm{SO}(1,3)$ components reads

$\mathbb{F}^{I J}=R^{I J}-\lambda^{2} e^{I} e^{J}, \quad\left(R^{I J}=d \omega^{I J}+\omega^{I}{ }_{K} \omega^{K J}\right)$

$\mathbb{F}^{I 4}=\lambda D e^{I} \quad\left(D e^{I}=d e^{I}+\omega^{I}{ }_{J} e^{J}\right)$.

The infinitesimal (A) $\mathrm{dS}_{5}$ gauge transformations which leave the Chamseddine action invariant may be written as

$\delta \mathbb{A}^{A B}=d \epsilon^{A B}+\mathbb{A}^{A}{ }_{C} \epsilon^{C B}-\mathbb{A}^{B}{ }_{C} \epsilon^{C A}$,

$\delta \Phi^{A}=\Phi_{B} \epsilon^{B A}$.

The equations of motion from (2.22) are

$$
\begin{aligned}
\frac{\delta S^{(4 D)}}{\delta \Phi^{A}} & =\frac{1}{8} \varepsilon_{A B C D E} \mathbb{F}^{B C} \mathbb{F}^{D E}=0, \\
\frac{\delta S^{(4 D)}}{\delta A^{A B}} & =\frac{1}{2} \varepsilon_{A B C D E} \mathbb{D} \Phi^{C} \mathbb{F}^{D E}=0 .
\end{aligned}
$$

\footnotetext{
${ }^{7}$ No parameter is needed in front of the action, since any such parameter may be absorbed in a redefinition of the scalar field $\Phi^{A}$.
} 
Or, in terms of the $\mathrm{SO}(1,3)$ components,

$$
\begin{aligned}
\frac{\delta S}{\delta e^{I}}= & -\frac{\lambda}{2} \varepsilon_{I J K L}\left(D \Phi^{J}+s \lambda e^{J} \Phi^{4}\right)\left(R^{K L}-s \lambda^{2} e^{K} e^{L}\right)=0 \\
\frac{\delta S}{\delta \omega^{I J}}= & \frac{1}{2} \varepsilon_{I J K L} \\
& \times\left(\left(d \Phi^{4}-\lambda e_{I^{\prime}} \Phi^{I^{\prime}}\right)\left(R^{K L}-s \lambda^{2} e^{K} e^{L}\right)\right. \\
& \left.+\lambda\left(D \Phi^{K}+s \lambda e^{K} \Phi^{4}\right) D e^{L}\right)=0, \\
\frac{\delta S}{\delta \Phi^{4}}= & \frac{1}{8} \varepsilon_{I J K L}\left(R^{I J}-s \lambda^{2} e^{I} e^{J}\right)\left(R^{K L}-s \lambda^{2} e^{K} e^{L}\right)=0 \\
\frac{\delta S}{\delta \Phi^{I}}= & \frac{\lambda}{2} \varepsilon_{I J K L} D e^{J}\left(R^{K L}-s \lambda^{2} e^{K} e^{L}\right)=0 .
\end{aligned}
$$

\subsubsection{Introducing matter: continuity equation}

We may introduce matter adding to the action (2.22) a matter term $S_{\mathrm{m}}$ which we will suppose $(\mathrm{A}) \mathrm{dS}_{5}$ gauge invariant and independent of the scalar field $\Phi^{A}$. (A) $\mathrm{dS}_{5}$ gauge invariance of the total action,

$S=S^{(4 D)}[e, \omega, \Phi]+S_{\mathrm{m}}[e, \omega]$,

can be expressed through the local "Ward identity"

$W_{A B} S:=-\mathbb{D} \frac{\delta S}{\delta \mathbb{A}}+\Phi_{A} \frac{\delta S}{\delta \Phi^{B}}-\Phi_{B} \frac{\delta S}{\delta \Phi^{A}}=0$.

We shall be interested in particular in the Ward identity linked to invariance along the generators $M_{I 4}$ :

$W_{I} S:=-\lambda e^{J} \frac{\delta S}{\delta \omega^{I J}}-\frac{1}{\lambda} D \frac{\delta S}{\delta e^{I}}+\Phi_{I} \frac{\delta S}{\delta \Phi^{4}}-s \Phi^{4} \frac{\delta S}{\delta \Phi^{I}}=0$.

Note that these identities hold separately for both actions, $S^{(4 D)}$ and $S_{\mathrm{m}}$. Defining

$\mathcal{T}_{I}:=\frac{\delta S_{\mathrm{m}}}{\delta e^{I}}, \quad \mathcal{T}_{I J}:=\frac{\delta S_{\mathrm{m}}}{\delta \omega^{I J}}$,

we may rewrite (2.29) as

$$
\begin{gathered}
\lambda e^{J} \mathcal{T}_{I J}+\frac{1}{\lambda} D \mathcal{T}_{I}=-\lambda e^{J} \frac{\delta S^{(4 D)}}{\delta \omega^{I J}}-\frac{1}{\lambda} D \frac{\delta S^{(4 D)}}{\delta e^{I}} \\
+\Phi_{I} \frac{\delta S^{(4 D)}}{\delta \Phi^{4}}-s \Phi^{4} \frac{\delta S^{(4 D)}}{\delta \Phi^{I}}=W_{I} S^{(4 D)}=0,
\end{gathered}
$$

the last equality expressing the invariance of $S^{(4 D)}$. This leads to the general continuity equation

$$
\lambda 2 e^{J} \mathcal{T}_{I J}+D \mathcal{T}_{I}=0
$$

The 3-form $\mathcal{T}_{I}$ is related to the energy-momentum tensor components $\mathcal{T}^{N}{ }_{I}$ in the tetrad frame by

$$
\mathcal{T}_{I}=\frac{1}{6} \varepsilon_{N J K L} \mathcal{T}^{N}{ }_{I} e^{J} e^{K} e^{L} .
$$

If $\mathcal{T}_{I J}=0$, i.e., if the matter action $S_{\mathrm{m}}$ does not depend on the spin connection $\omega,(2.31)$ is interpreted as the continuity equation for energy and momentum.

\subsubsection{Partial gauge fixing of the Chamseddine theory}

From the gauge transformations (2.24) leaving the action (2.22) invariant, one sees that a possible partial gauge fixing is given by the four conditions

$\Phi^{I}=0, \quad I=0, \ldots, 3$.

The total action, including matter, then reduces to

$$
\begin{aligned}
\bar{S}= & \frac{1}{8} \int_{\mathcal{M}_{4}} \varepsilon_{I J K L} \Phi^{4} \mathbb{F}^{I J} \mathbb{F}^{K L}+S_{\mathrm{m}} \\
= & \frac{1}{8} \int_{\mathcal{M}_{4}} \varepsilon_{I J K L} \Phi^{4}\left(R^{I J}-s \lambda^{2} e^{I} e^{J}\right) \\
& \left(R^{K L}-s \lambda^{2} e^{K} e^{L}\right)+S_{\mathrm{m}},
\end{aligned}
$$

where the matter action $S_{\mathrm{m}}$ is supposed to be independent of $\Phi^{A}$, as above, but will also be assumed not to depend on the spin connection $\omega$ from now on. The field equations derived from the latter action - to which we may add a matter action $S_{\mathrm{m}}$, supposed to obey the same (A) $\mathrm{dS}_{5}$ gauge invariance as the pure Chamseddine part—are

$$
\begin{aligned}
\frac{\delta \bar{S}}{\delta e^{I}} & =-\frac{1}{2} s \lambda^{2} \Phi^{4} \epsilon_{I J K L}\left(e^{J} R^{K L}-s \lambda^{2} e^{J} e^{K} e^{L}\right)+\mathcal{T}_{I}=0, \\
\frac{\delta \bar{S}}{\delta \omega^{I J}} & =\frac{1}{2} \epsilon_{I J K L}\left(\mathrm{~d} \Phi^{4}\left(R^{K L}-s \lambda^{2} e^{K} e^{L}\right)+2 s \lambda^{2} \Phi^{4} e^{K} D e^{L}\right)=0, \\
\frac{\delta \bar{S}}{\delta \Phi^{4}} & =\frac{1}{8} \epsilon_{I J K L}\left(R^{I J}-s \lambda^{2} e^{I} e^{J}\right)\left(R^{K L}-s \lambda^{2} e^{K} e^{L}\right)=0,
\end{aligned}
$$

where $T^{I}:=D e^{I}$ is the torsion and $\mathcal{T}_{I}$ the energymomentum 3-form (2.32).

Comparison of the first of Eq. (2.35) with the standard Einstein equation in the first order formalism,

$$
\begin{aligned}
& \varepsilon_{I J K L}\left(e^{J} R^{K L}-\frac{\Lambda}{3} e^{J} e^{K} e^{L}\right)=-16 \pi G \mathcal{T}_{I}, \\
& T^{I}=D e^{I}=0
\end{aligned}
$$

suggests one to identify $3 s \lambda^{2}$ with the cosmological constant,

$\Lambda:=3 s \lambda^{2}$,

and to define the function

$G(x):=-\frac{3}{8 \pi \Lambda \Phi^{4}(x)}$,

as a variable "Newton parameter", proportional to the inverse of the dilation field $\Phi^{4}$. With this, the field equations (2.35) take the form 


$$
\begin{aligned}
& \epsilon_{I J K L}\left(e^{J} R^{K L}-\frac{\Lambda}{3} e^{J} e^{K} e^{L}\right)=-16 \pi G(x) \mathcal{T}_{I}, \\
& \epsilon_{I J K L}\left(\mathrm{~d} G(x)\left(R^{K L}-\frac{\Lambda}{3} e^{K} e^{L}\right)-2 \frac{\Lambda}{3} G(x) e^{K} D e^{L}\right)=0, \\
& \epsilon_{I J K L}\left(R^{I J}-\frac{\Lambda}{3} e^{I} e^{J}\right)\left(R^{K L}-\frac{\Lambda}{3} e^{K} e^{L}\right)=0
\end{aligned}
$$

where we have emphasized the $x$-dependence of the Newton parameter $G$.

Let us finish this subsection with some comments:

1. The theory is clearly singular in $\Lambda=0$. This value would correspond to a vanishing $s$ in the (A) $\mathrm{dS}_{5}$ metric (A.2), which thus would become singular.

2. The first field equation in (2.35) has the form of the usual Einstein equation in the first order formalism, but with varying Newton coupling parameter $G(x)$. The second one determines the torsion $T^{I}$ in terms of the basic fields $\omega^{I J}, e^{I}$, and $G$. In particular, the torsion is zero if $G$ is constant. The third equation is a new constraint. One must emphasize that the torsion here is not an independent field.

3. A canonical analysis [19] shows that the number of physical degrees of freedom of the theory is three: this corresponds to the two degrees of freedom of the gravitational field plus one corresponding to the scalar dilaton field $\Phi^{4}$ — the Newton coupling parameter $G$.

4. In the absence of matter, an obvious trivial solution is the constant curvature and torsion free (anti-)de Sitter space: $R^{I J}=\frac{\Lambda}{3} e^{I} e^{J}$.

5. The last equation, which clearly admits the constant curvature solution, is also compatible with non-trivial solutions, as the examples treated below do show.

6. It is interesting to note that the gauge fixing condition (2.33) is nothing but the first of the gauge fixing conditions (2.17) [see Definition (2.21)].

\subsubsection{Energy-momentum continuity}

In Einstein theory, the continuity equation for the energymomentum tensor reads, in the first order formalism used here,

$D \mathcal{T}_{I}=0$

where $D$ is the exterior derivative with respect to the spin connection $\omega^{I J}$ and the 3 -form $\mathcal{T}_{I}$ is related to the energymomentum tensor by (2.32). The continuity equation (2.40) follows from the Einstein field equations (2.36) and the Bianchi identity $D R^{I J}=0$. As we saw in Sect. (2.2.1), it turns out that it still holds in our case, as a consequence of the (A) $\mathrm{dS}_{5}$ invariance expressed by the identity (2.30) and of the hypothesis we have made that the matter action is independent not only from the scalar fields $\Phi$, but also from the spin connection $\omega$.

It is interesting to look at the identity (2.30) with the gauge fixing condition $\Phi^{I}=0$ being applied. Taking into account the hypothesis that $S_{\mathrm{m}}$ only depends on the tetrad $e$, this leads to the identity

$D \mathcal{T}_{I}=\frac{\Lambda}{3} e^{J} \frac{\delta \bar{S}}{\delta \omega^{I J}}+\left.\sqrt{\frac{|\Lambda|}{3}} \Phi^{4} \frac{\delta S}{\delta \Phi^{I}}\right|_{\Phi^{I}=0}$

where $\bar{S}$ is the total gauge fixed action (2.34), and $S$ the total action (2.28) before gauge fixing. Since $D \mathcal{T}_{I}=0$, the latter identity shows that the equation

$$
\left.\frac{\delta S}{\delta \Phi^{I}}\right|_{\Phi^{I}=0}=0
$$

is valid "on shell", i.e., if the field equations (2.35) of the gauge fixed theory are satisfied. This is just the equation of the non-gauge fixed theory obtained by varying $\Phi^{I}$, taken at $\Phi^{I}=0$. In fact, the on-shell validity of (2.41) can be derived directly from the Ward identity (2.29) taken at $\Phi^{I}=0$, as one can easily check.

\subsubsection{The field equations of the Chamseddine model as particular field equations of the dimensionally reduced 5D Chern-Simons theory}

One may ask if the equations of motion derived from the truncated theory, namely the Chamseddine model equations (2.25) and (2.26), together with the truncation equations (2.19) and the $\chi$-independence conditions (2.18), are also solutions of the equations of motion (2.5) of the full original (A) $\mathrm{dS}_{6}$ Chern-Simons theory. In the following we show the answer is positive.

The field equations of the full CS theory reduced in four dimensions are given by (2.14) and (2.15) together with (2.13). After imposing the truncation (2.19) together with the restriction (2.18) and the relabeling (2.20), (2.21), the curvature components take the form

$$
\begin{aligned}
& F^{I J}=R^{I J}-s \lambda^{2} e^{I} e^{J}, \quad F^{I 4}=0, \quad F^{I 5}=\lambda D e^{I}, \quad F^{45}=0, \\
& F_{\chi}^{I J}=0, \quad F_{\chi}^{I 4}=-\lambda D \Phi^{I}-s \lambda^{2} e^{I} \Phi^{4}, \quad F_{\chi}^{I 5}=0 \\
& F_{\chi}^{45}=\lambda d \Phi^{4}-\lambda^{2} e_{I} \Phi^{I} .
\end{aligned}
$$

Inserting the expressions (2.42) in the eight equations (2.14) and (2.15), we obtain four trivial equations $0=0$, and four non-trivial ones which are identical to those obtained from the action of the $(\mathrm{A}) \mathrm{dS}_{5}$ Chamseddine model, Eq. 2.27. We conclude that the set of solutions of the equations of motion of the $(\mathrm{A}) \mathrm{dS}_{5}$ is a particular subset of the solutions of the general (A) $\mathrm{dS}_{6}$ Chern-Simons theory. 
It is noteworthy that the four trivial equations are those derived from the CS action by varying the four fields destined for truncation. Had we performed any other sort of truncation in the field equations, we would have obtained more independent equations than what one obtains directly from the truncated action.

It is also enlightening to see that the effect of the truncation, when applied directly to the original $(\mathrm{A}) \mathrm{d} \mathrm{S}_{6}$ connection, leads to

$\hat{A}=\frac{1}{2} \mathbb{A}_{\mu}^{A B} M_{A B} \mathrm{~d} x^{\mu}+\Phi^{A} P_{A} \mathrm{~d} \chi$,

where $\mathbb{A}^{A B}$ and $\Phi^{A}$ were introduced in (2.20) and (2.21). We see that the effect of the truncation is to confine the (A) $\mathrm{dS}_{5}$ symmetry to a four-dimensional connection, whereas the "translational" sector of the group is restricted to the $\chi$ dimension. Then it is clear why the truncation, which may seem not obvious at first sight, results after some simplifications in a four-dimensional (A) $\mathrm{dS}_{5}$ gauge theory with $\Phi$ a four-dimensional scalar transforming as a vector under (A) $\mathrm{dS}_{5}$ transformations.

The curvature associated with the truncated connection (2.43) is

$\hat{F}=\frac{1}{4} \mathbb{F}_{\mu \nu}^{A B} M_{A B} \mathrm{~d} x^{\mu} \mathrm{d} x^{\nu}+\mathbb{D}_{\mu} \Phi^{A} P_{A} \mathrm{~d} x^{\mu} \mathrm{d} \chi$.

A straightforward calculation shows that by replacing the above result in (2.5) we obtain $(2.25),(2.26)$, the field equations of the truncated action (2.22).

\section{Linear approximations}

In order to investigate the Newtonian limit of the Chamseddine theory or to look for the presence of wave-like solutions of the theory in the vacuum, we split the field variables between background ones, marked with an index ${ }^{0}$ on the top, and perturbations as follows:

$\omega^{I J}=\stackrel{\circ}{\omega}^{I J}+a^{I J}, \quad e^{I}=\stackrel{\circ}{e}^{I}+h^{I}, \quad G=\stackrel{\circ}{G}+\phi$.

Up to terms of order higher than one in the perturbation, the curvature $R=\mathrm{d} \omega+\omega^{2}$ and the torsion $T^{I}=D e^{I}$ read

$R^{I J}=\stackrel{\circ}{R}^{I J}+\stackrel{\circ}{D} a^{I J}, \quad T^{I}=\stackrel{\circ}{T}^{I}+\stackrel{\circ}{D} h^{I}+a^{I}{ }_{J} \stackrel{\circ}{ }^{J}$,

where $\stackrel{\circ}{D}$ is the covariant derivative corresponding to the background connection $\stackrel{\circ}{\omega}$. The background considered here is a constant curvature de Sitter space-time, solution of

$\stackrel{\circ}{R}^{I J}-\frac{\Lambda}{3} \stackrel{\circ}{ }^{I} \stackrel{\circ}{e}=0$,

hence the expression $F^{I J}=R^{I J}-\frac{\Lambda}{3} e^{I} e^{J}$ is of first order:

$$
\begin{aligned}
& F^{I J}=f^{I J}(+ \text { orders }>1), \\
& f^{I J}=\stackrel{\circ}{D} a^{I J}-\frac{\Lambda}{3}\left(\stackrel{\bullet}{e}^{I} h^{J}-\stackrel{\ominus}{e}^{J} h^{I}\right) .
\end{aligned}
$$

We shall also assume that the zeroth order Newton parameter $\stackrel{\circ}{G}$ is a (non-zero) constant. The second field equation then implies that the zeroth order torsion is vanishing: $\stackrel{\circ}{T}^{I}=0$. The first field equation shows that the energy-momentum 3form $\mathcal{T}_{I}$ must be considered to be of first order, and the third equation is identically solved up to and including the first order.

The first and second field equations read, at first order,

$$
\begin{aligned}
& \varepsilon_{I J K L} \stackrel{\circ}{ }^{J}\left(\stackrel{\circ}{D} a^{K L}-\frac{\Lambda}{3}\left(\stackrel{\ominus}{e}^{K} h^{L}-\stackrel{\ominus}{e}^{L} h^{K}\right)\right)=-8 \pi G_{0} \mathcal{T}_{I}, \\
& \varepsilon_{I J K L} G_{0} \stackrel{\circ}{ }^{K}\left(\stackrel{\circ}{D} h^{I}+a_{J}^{I} \stackrel{\circ}{ }^{J}\right)=0 \text {, }
\end{aligned}
$$

where $G_{0}=\stackrel{\circ}{G}$ is the Newton parameter at zeroth order, interpreted as the actual Newton constant. The second of these equations implies a vanishing torsion at first order, too:

$\stackrel{\circ}{D} h^{I}+a^{I}{ }_{J} \stackrel{\circ}{J}^{J}=0$.

We are thus left with the first of equations (3.2), where the first order connection $a^{I J}$ may be solved in terms of the vierbein perturbation components $h_{\mu}^{I}$ and their derivatives through the null torsion condition. This is just Einstein GR with cosmological constant at first order of the perturbation, in a de Sitter background.

A first implication is that the theory admits a Newtonian limit like Einstein's does. A second implication concerns the theory with cosmological constant in the vacuum. Since at first order the theory coincides with Einstein's, we can rely on the results of an extensive study made by the authors of [20, 21], where they show that, beyond the constant curvature solution, there are propagating wave solutions. We refer to their paper for more details.

\section{Cosmological solutions}

In order to explore the physical content of the Chamseddine model, we look in this section for solutions of the cosmological type and compare them with the known $\triangle \mathrm{CDM}$ results [22].

\subsection{Isotropy and homogeneity}

We examine the solutions of the field equations (2.35) considering a space-time foliated by a family of isotropic and homogeneous three-dimensional spatial slices, as described by the standard Big Bang cosmology. The metric that describes this is the Friedmann-Lemaître-Robertson-Walker (FLRW) metric, given by

$$
\mathrm{d} s^{2}=-\mathrm{d} t^{2}+a^{2}(t)\left[\frac{\mathrm{d} r^{2}}{1-k r^{2}}+r^{2} \mathrm{~d} \theta^{2}+r^{2} \sin ^{2} \theta \mathrm{d} \varphi^{2}\right],
$$


depending on the time-dependent scale factor $a(t)$ and the space curvature parameter $k=0, \pm 1$. The space-time coordinates are the time coordinate $t$ and the spatial spherical coordinates $r, \theta, \varphi$. The FLRW metric admits six isometries generated by six global Killing vectors associated with three spatial translation $\xi_{(a)}$ and three rotation $\xi_{[a b]}$ invariancesi.e., such that $\mathcal{L}_{\xi_{[a b]}} g_{\mu \nu}=\mathcal{L}_{\xi_{(a)}} g_{\mu \nu}=0$-which read in Cartesian coordinates $x^{a}, a=1,2,3$,

$\xi_{(a)}=\sqrt{1-k r^{2}} \partial_{a}$, and $\xi_{[a b]}=x_{a} \partial_{b}-x_{b} \partial_{a}$.

We assume that the torsion and the scalar field (the Newton parameter $G$ ) have the same isometries as the metric, i.e., $\mathcal{L}_{\xi} T^{\rho}{ }_{\mu \nu}=0$ and $\mathcal{L}_{\xi} G=0$. These conditions imply $G=$ $G(t)$, and the non-vanishing components of $T^{\alpha}{ }_{\mu \nu} \operatorname{are}^{8}$ [23]

$T^{r}{ }_{\theta \varphi}=2 f(t) a(t) r^{2} \sqrt{1-k r^{2}} \sin \theta, \quad T_{r \theta}^{\varphi}=\frac{2 f(t) a(t)}{\sqrt{1-k r^{2}} \sin \theta}$,

$T_{r \varphi}^{\theta}=-\frac{2 f(t) a(t) \sin \theta}{\sqrt{1-k r^{2}}}, \quad T^{r}{ }_{r t}=T^{\theta} \theta_{\theta t}=T_{\varphi t}^{\varphi}=h(t)$

where $f(t)$ and $h(t)$ are functions of time to be determined by the field equations.

Working in the first order formalism, we have to choose a corresponding parametrization of the vierbein. A convenient choice [23] is ${ }^{9}$ :

$e^{0}=\mathrm{d} t, \quad e^{1}=\frac{a(t)}{\sqrt{1-k r^{2}}} \mathrm{~d} r$

$e^{2}=a(t) r \mathrm{~d} \theta, \quad e^{3}=a(t) r \sin \theta \mathrm{d} \varphi$.

In this basis the torsion 2-form becomes

$T^{0}=0, \quad T^{i}=h(t) e^{i} e^{0}+f(t) \varepsilon^{i}{ }_{j k} e^{j} e^{k}$.

(The indices $i, j \ldots$ take the values $1,2,3$.) The spin connection $\omega$ which gives rise to this torsion reads

$\omega^{0 i}=(H+h) e^{i}, \quad \omega^{12}=-\frac{\sqrt{1-k r^{2}}}{a r} e^{2}-f e^{3}$,

$\omega^{31}=\frac{\sqrt{1-k r^{2}}}{a r} e^{3}-f e^{2}, \quad \omega^{23}=-\frac{\cot \theta}{a r} e^{3}-f e^{1}$,

where

$H:=\dot{a}(t) / a(t)$

is the Hubble parameter. The Riemann curvature is given by

$R^{0 i}=((\dot{H}+\dot{h})+H(H+h)) e^{0} e^{i}+f(H+h) \varepsilon_{j k}^{i} e^{j} e^{k}$,

$R^{i j}=\left((H+h)^{2}+\frac{k}{a^{2}}-f^{2}\right) e^{i} e^{j}+(\dot{f}+H f) \varepsilon^{i j}{ }_{k} e^{k} e^{0}$.

$\overline{8 \text { Torsion } T^{I}{ }_{\mu \nu}}$ is defined by $T^{I}=D e^{I}$, whereas $T^{\rho}{ }_{\mu \nu}=e_{I}^{\rho} T^{I}{ }_{\mu \nu}$.

9 This choice amounts to a gauge fixing of the local Lorentz invariance.
Consequently

$$
\begin{aligned}
F^{0 i}= & \left((\dot{H}+\dot{h})+H(H+h)-\frac{\Lambda}{3}\right) e^{0} e^{i} \\
& +f(H+h) \varepsilon^{i}{ }_{j k} e^{j} e^{k}, \\
F^{i j}= & \left((H+h)^{2}+\frac{k}{a^{2}}-f^{2}-\frac{\Lambda}{3}\right) e^{i} e^{j} \\
& +(\dot{f}+H f) \varepsilon^{i j}{ }_{k} e^{k} e^{0} .
\end{aligned}
$$

\subsection{Field equations}

We assume matter to consist of a perfect fluid of density $\rho_{\mathrm{m}}$ and pressure $p_{\mathrm{m}}$, with an energy-momentum tensor $\mathcal{T}^{I}{ }_{J}=$ diag $\left(-\rho_{\mathrm{m}}, p_{\mathrm{m}}, p_{\mathrm{m}}, p_{\mathrm{m}}\right)$. Substituting in the field equations (2.35), with $d G=\dot{G} e^{0}$, we get the system of differential equations

$$
\begin{aligned}
& U^{2}+\frac{k}{a^{2}}-f^{2}-\frac{\Lambda}{3}=\frac{8 \pi G}{3} \rho_{\mathrm{m}}, \\
& U^{2}+\frac{k}{a^{2}}-f^{2}-\Lambda+2(\dot{U}+H U)=-8 \pi G p_{\mathrm{m}}, \\
& \dot{G}\left(U^{2}+\frac{k}{a^{2}}-f^{2}-\frac{\Lambda}{3}\right)-\frac{2 \Lambda}{3} G h=0, \\
& f\left(\dot{G} U-\frac{\Lambda}{3} G\right)=0, \\
& \left(U^{2}+\frac{k}{a^{2}}-f^{2}-\frac{\Lambda}{3}\right)\left(\dot{U}+H U-\frac{\Lambda}{3}\right) \\
& \quad-2 f U(\dot{f}+H f)=0,
\end{aligned}
$$

where $U:=H+h$ and $G=G(t)$ is the Newton coupling parameter (2.38).

\subsection{Continuity equations}

A first continuity equation for the energy and pressure of matter follows directly from the energy-momentum continuity equation (2.40). Calculating the components of the energymomentum 3-form $\mathcal{T}_{I}$, from (2.32) one finds

$\mathcal{T}_{0}=-\frac{\rho_{m}(t)}{6} \epsilon_{i j k} e^{i} e^{j} e^{k}$,
$\mathcal{T}_{i}=-\frac{p_{m}(t)}{2} \epsilon_{i j k} e^{0} e^{j} e^{k}$,

consequently

$\mathcal{T}_{0}=-\rho_{m}(t) e^{1} e^{2} e^{3}=-\frac{\rho_{m}(t) a(t)^{3} r^{2} \sin \theta}{\sqrt{1-k r^{2}}} \mathrm{~d} r \wedge \mathrm{d} \theta \wedge \mathrm{d} \varphi$,

$\mathcal{T}_{1}=-p_{m}(t) e^{0} e^{2} e^{3}=-p_{m}(t) a(t)^{2} r^{2} \sin \theta \mathrm{d} t \wedge \mathrm{d} \theta \wedge \mathrm{d} \varphi$,

$\mathcal{T}_{2}=-p_{m}(t) e^{0} e^{3} e^{1}=-p_{m}(t) \frac{a(t)^{2} r \sin \theta}{\sqrt{1-k r^{2}}} \mathrm{~d} t \wedge \mathrm{d} \varphi \wedge \mathrm{d} r$,

$\mathcal{T}_{3}=-p_{m}(t) e^{0} e^{1} e^{2}=-p_{m}(t) \frac{a(t)^{2} r}{\sqrt{1-k r^{2}}} \mathrm{~d} t \wedge \mathrm{d} r \wedge \mathrm{d} \theta$. 
The equation $D \mathcal{T}_{0}=0$ yields the density-pressure-torsion continuity equation

$\dot{\rho}_{\mathrm{m}}+3 H\left(p_{\mathrm{m}}+\rho_{\mathrm{m}}\right)+3 h p_{\mathrm{m}}=0$.

The equations $D \mathcal{T}_{i}=0$ for $i=1,2,3$ are trivially satisfied, being of the form $0=0$.

Note the torsion dependence in the last term of (4.7). However, for a matter with zero pressure (cold matter, dust), this continuity equation takes the usual form [24]:

$\frac{\mathrm{d}}{\mathrm{d} t}\left(\rho_{\mathrm{m}} a^{3}\right)=0, \quad$ if $p_{\mathrm{m}}=0$.

A second continuity equation can be found in the following way: One notes that, substituting $U=H+h$ in Eqs. (4.2) and (4.3) leads to analogs of the standard Friedmann equations:

$H^{2}=\frac{8 \pi G_{0}}{3} \rho_{\text {tot }}, \quad 2 \dot{H}+3 H^{2}=-8 \pi G_{0} p_{\text {tot }}$,

where $G_{0}$ is the Newton constant, taken as the present value of $G(t)$, and $\rho_{\text {tot }}, p_{\text {tot }}$ are the "total density and pressure",

$\rho_{\mathrm{tot}}=\frac{G}{G_{0}}\left(\rho_{\mathrm{m}}+\rho_{k}+\rho_{T}+\rho_{\Lambda}\right)$,

$p_{\text {tot }}=\frac{G}{G_{0}}\left(p_{\mathrm{m}}+p_{k}+p_{T}+p_{\Lambda}\right)$,

with

$\rho_{k}=-\frac{3}{8 \pi G} \frac{k}{a^{2}} \quad \rho_{T}=\frac{3}{8 \pi G}\left(f^{2}-2 H h-h^{2}\right), \quad \rho_{\Lambda}=\frac{\Lambda}{8 \pi G}$

$p_{k}=-\rho_{k} / 3, \quad p_{T}=\frac{1}{8 \pi G}\left(2 \dot{h}+4 H h+h^{2}-f^{2}\right), \quad p_{\Lambda}=-\rho_{\Lambda}$.

$\rho_{T}$ and $p_{T}$ may be interpreted as the contributions of the torsion to the total density and pressure $\rho_{\text {tot }}$ and $p_{\text {tot }}$. As a consequence of the Friedmann-like equations (4.9), the total density and pressure satisfy the continuity equation

$\dot{\rho}_{\text {tot }}+3 H\left(\rho_{\text {tot }}+p_{\text {tot }}\right)=0$.

\subsection{Pressure-less matter with $\Lambda>0$ and $k=0$}

In this subsection we present the general solution of Eqs. (4.2)-(4.6) in the case of pressure-less matter (cold matter or dust), with $p_{\mathrm{m}}=0$, with a positive cosmological constant $\Lambda$ and a null curvature parameter $k$, as favored by the observational results [22]. From Eq. (4.5) follows

either $f(t)=0, \quad$ or $\quad \dot{G} U-\frac{\Lambda}{3} G=0$.

We have first checked that the former case leads to the "trivial" solution of a null torsion de Sitter space with cosmological constant $\Lambda$, the vierbein or the metric being defined by the scale parameter $a(t)=\exp (\sqrt{\Lambda / 3} t)$.

We hence assume the function $f(t)$ to be non-vanishing. The equations to be solved are Eqs. (4.2)-(4.4), (4.6), and the second of (4.10), together with the Hubble parameter definition (4.1) in terms of the scale $a(t)$. The general solution is given by the following expressions ${ }^{10}$, where the time coordinate has been redefined by

$\tau(t):=\sqrt{\frac{\Lambda}{3}} t$.

Scale parameter:

$a(t)=C_{4}\left(3 e^{\tau(t)}+C_{3} e^{-\tau(t)}\right)^{1 / 3}\left(\cosh \left(\tau(t)-C_{1}\right)\right)^{2 / 3}$.

Torsion parameter $f(t)$ :

$$
\begin{aligned}
f(t)= & \frac{\sqrt{\Lambda}}{3}\left[\left(-9 e^{2 \tau(t)}-3 C_{3}+\left(6 e^{2 \tau(t)}-2 C_{3}\right) \tanh \left(\tau(t)-C_{1}\right)\right.\right. \\
& \left.\left.+\left(3 e^{2 \tau(t)}+C_{3}\right) \tanh ^{2}\left(\tau(t)-C_{1}\right)\right) /\left(3 e^{2 \tau(t)}+C_{3}\right)\right]^{1 / 2} .
\end{aligned}
$$

\section{Torsion parameter $h(t)$ :}

$h(t)=\sqrt{\frac{\Lambda}{3}} \frac{\left(-3 e^{2 \tau(t)}+C_{3}+\left(3 e^{2 \tau(t)}+C_{3}\right) \tanh \left(\tau(t)-C_{1}\right)\right)}{9 e^{2 \tau(t)}+3 C_{3}}$.

Hubble parameter $H=\dot{a} / a$ :

$H(t)=\sqrt{\frac{\Lambda}{3}} \tanh \left(\tau(t)-C_{1}\right)-h[t]$.

Newton parameter $G(t)=-3 /(8 \pi \Lambda \Phi(t))[c f .(2.38)]$ :

$G(t)=C 2 \sinh \left(\tau(t)-C_{1}\right)$.

Cold matter density:

$\rho_{\mathrm{m}}(t)=\frac{3}{8 \pi G(t)}\left((H(t)+h(t))^{2}-f^{2}(t)-\frac{\Lambda}{3}\right)$.

The four integration constants $C_{1}, C_{2}, C_{3}, C_{4}$ and the cosmological constant $\Lambda$ have to be determined by five physical conditions, which we choose to be

$a(0)=0:$ hypothesis of a Big Bang,

$a\left(t_{0}\right)=1: t_{0}=$ present age of the Universe,

$H\left(t_{0}\right)=H_{0}:$ present value of the Hubble parameter,

$G\left(t_{0}\right)=G_{0}:$ present value of the Newton parameter,

$\rho_{\mathrm{m}}\left(t_{0}\right)=\rho_{0}: \quad$ present value of the cold matter mass density,

with the present observational [22] and experimental data given by

$$
\begin{aligned}
t_{0} & =13.8 \times 10^{9} \mathrm{~Gy} \quad\left(1 \mathrm{~Gy}=10^{9} \text { years }=3.1558^{16} \mathrm{~s}\right), \\
H_{0} & =0.0693 \mathrm{~Gy}^{-1}, \\
\rho_{0} & =2.664 \times 10^{-27} \mathrm{Kg} \mathrm{m}^{-3},
\end{aligned}
$$

$\overline{10}$ The solution is obtained using the program Mathematica [25]. 
$G_{0}=6.674 \times 10^{-11} \mathrm{~m}^{3} \mathrm{~s}^{-2} \mathrm{~kg}^{-1}$.

For comparison with the standard $\Lambda \mathrm{CDM}$ results, we need the $\Lambda C D M$ formula for the scale parameter $a(t)$, for a Universe dominated by cold dark matter of the present relative density [22] $\Omega_{\mathrm{m}}=0.309$. With the contribution of radiation neglected, the normalized $\Lambda$ CDM scale parameter reads [24]

$$
\begin{aligned}
& a_{\Lambda \mathrm{CDM}}(t) \\
& \quad=\left(\sinh \left(\frac{3}{2} H_{0} \sqrt{1-\Omega_{\mathrm{m} 0}} t\right) / \sinh \left(\frac{3}{2} H_{0} \sqrt{1-\Omega_{\mathrm{m} 0}} t_{0}\right)\right)^{2 / 3} .
\end{aligned}
$$

Figure 1 shows the time evolution of the scale parameter $a$, of the Hubble constant $H$, of the deceleration parameter $q$ $=-\ddot{a} a /\left(\dot{a} a^{2}\right)$, of the mass density $\rho_{\mathrm{m}}$, and of the normalized Newton parameter $G / G_{0}$, each one being compared with the corresponding $\Lambda \mathrm{CDM}$ quantity. Except for the deceleration $q$, the deviations are rather small. The Newton parameter, which has to be equal to the actual Newton constant $G_{0}$ at the present time, shows a slight decrease toward the past, growing to $\sim 85 \%$ of its present value near of the Big Bang. The deceleration $q$ differs notably from the $\Lambda \mathrm{CDM}$ one, but the time of the transition between the deceleration and the acceleration era almost coincides. The present value $q\left(t_{0}\right)=$ -0.25 is, however, only half of the $\Lambda \mathrm{CDM}$ value.

Time evolutions of the torsion parameters $h$ and $f$, as well as the relative densities $\Omega_{\mathrm{m}}(t), \Omega_{\Lambda}(t)$, and $\Omega_{T}(t)$ for matter, cosmological constant, and torsion, respectively, are shown in Fig. 2a, b.
We observe from Fig. $2 \mathrm{c}$ that the end of the cold matter dominance area, at $t \sim 10.2$ Gy for $\Lambda \mathrm{CDM}$, occurs at $t \sim 8.5$ Gy for our model, matter dominance being defined, in the latter case, as the dominance of $\Omega_{\mathrm{m}}$ over the sum $\Omega_{\Lambda}+\Omega_{T}$.

Finally, the present values of the concentrations are:

- $\Omega_{\mathrm{m}}\left(t_{0}\right)=0.308$ (which belongs to the input data),

- $\Omega_{\Lambda}\left(t_{0}\right)=0.289$,

- $\Omega_{T}\left(t_{0}\right)=0.403$.

This has to be contrasted with the $\Lambda \mathrm{CDM}$ values $\Omega_{\mathrm{m}}\left(t_{0}\right)=$ 0.308 and $\Omega_{\Lambda}\left(t_{0}\right)=0.692$ : in our model the torsion contributes together with the cosmological constant to the acceleration.

Finally, as a matter of verification, we have checked that our solution of the field equations does satisfy the continuity equation (4.8).

\subsection{Search for other solutions}

Since torsion may contribute to the acceleration, as in the solution studied above, one could expect solutions presenting a positive present acceleration even with a negative cosmological constant. This occurs for instance for the class of models investigated in [23]. In our case, we have checked that there is no solution with $\Lambda<0$ and a positive acceleration fulfilling the physical boundary conditions represented by the present values of the cold matter density and of the Hubble and Newton parameters. Another class of solutions with a bounce at some time in the past do exist, but none of
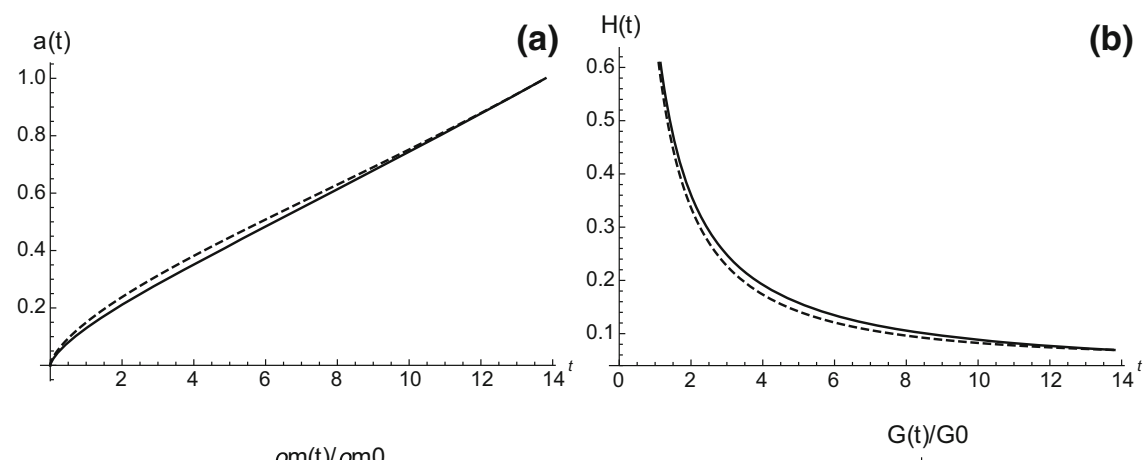

(b)

(c)
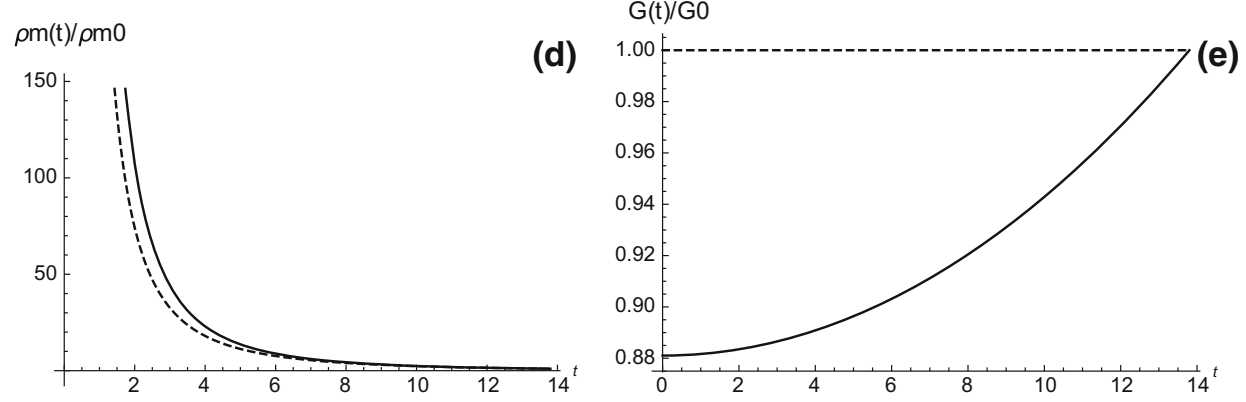

Fig. 1 a Normalized scale parameter $a(t)$; b Hubble parameter $H(t)$; c deceleration parameter $q(t)$; d cold matter density $\rho_{\mathrm{m}}(t)$; e time-dependent gravitation coupling parameter $G(t)$; Solid lines model predictions; dashed lines standard $\Lambda \mathrm{CDM}$ results 

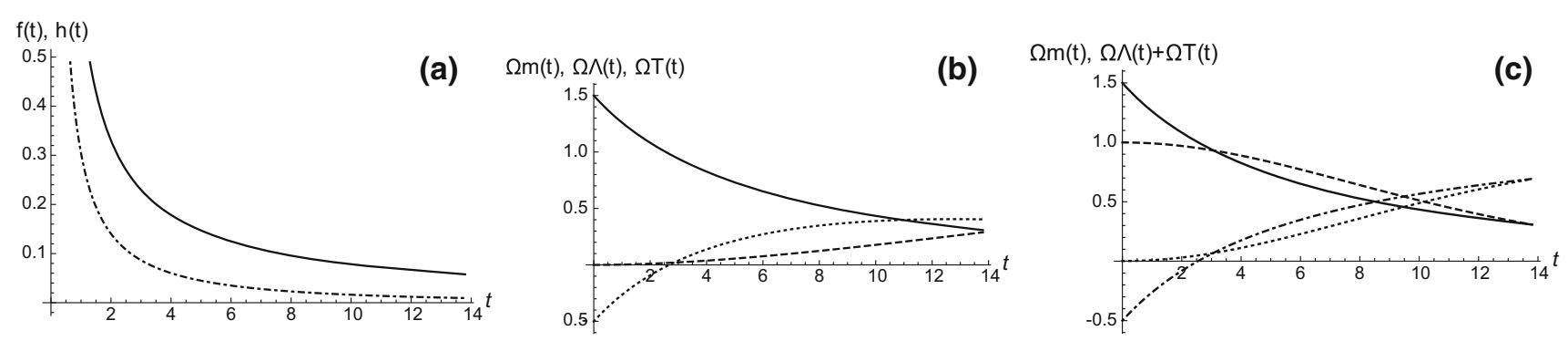

Fig. 2 a Torsion parameters $f(t)$ (solid line) and $h(t)$ (dashed line); b relative densities $\Omega_{\mathrm{m}}(t)$ (solid line), $\Omega_{\Lambda}(t)($ dashed line $)$ and $\Omega_{T}(t)($ dotted line); $\mathbf{c} \Omega_{\mathrm{m}}(t)$ (solid line)and $\Omega_{\Lambda}(t)+\Omega_{T}(t)$ (dot-dashed line); $\Lambda \mathrm{CDM}$ results are shown for $\Omega_{\mathrm{m}}$ (dashed line) and $\Omega_{\Lambda}($ dotted line)

them is compatible even roughly with the physical boundary conditions.

\section{Conclusion and outlooks}

We have seen in rather great detail how the dimensional reduction and truncation of the $(\mathrm{A}) \mathrm{dS}_{6}$ Chern-Simons theory in $5 \mathrm{D}$ to the $4 \mathrm{D}$ Chamseddine model is working. In particular, we have shown that the field equations of the latter form a subset of the field equations of the former, which is a non-trivial result. Chamseddine's theory involves a scalar dilaton-like field which we have interpreted as a varying Newton coupling parameter. We have explored the solutions of the field equations, focusing on two examples. In the first one we have shown the existence, in a linear approximation, of a Newtonian limit and of gravitational waves of the same type as the ones of standard GR. The Newton parameter is supposed to be constant in the zeroth order and turns out to remain undetermined at first order. The wave solutions confirm the canonical result [19] of three degrees of freedom: two for the "graviton" and one for the Newton parameter field. The second example is of the FLRW cosmological type. We have found a solution with boundary conditions corresponding to the present values of the physical parameters: the Newton and Hubble parameters, and the cold matter mass density. It shows a behavior fitting rather well that of the standard $\Lambda \mathrm{CDM}$ model, at least qualitatively. The cosmological constant of this solution turns out to be positive, however, smaller than that of the $\Lambda \mathrm{CDM}$ model, the torsion contributing substantially to the present acceleration of the expansion. A similar but different model has been studied by the authors of [23]. The main difference is that, in their action, a scalar field appears as a factor only in the supplementary term, quadratic in the curvature. In our case, the scalar field appears as a common factor of the whole Lagrangian density and, moreover, the term quadratic in the curvature is not independent due to the constraint of the $(\mathrm{A}) \mathrm{dS}_{5}$ gauge symmetry $\mathrm{SO}(1,4)$ or $\mathrm{SO}(2,3)$.

A study of the full 5D Chern-Simons theory is under way, with one spatial dimension being compactified [19]. It will allow one to explore a larger domain of solutions, this theory possessing 13 degrees of freedom as shown in $[17,18]$.
Concerning the quantization, the prospect [19] is for a Loop Quantization [1,2] of the 5D CS theory. Indeed, the latter is generic in the sense of the authors of $[17,18]$, i.e., the scalar or "Hamiltonian" constraint is a consequence of the other constraints, which are easier to solve $[1,2]$.

Acknowledgments This work was partially funded by the Fundação de Amparo à Pesquisa do Estado de Minas Gerais-FAPEMIG, Brazil (O.P.), the Conselho Nacional de Desenvolvimento Científico e Tecnológico-CNPq, Brazil (I.M., Z.O. and O.P.), and the Coordenação de Aperfeiçoamento de Pessoal de Nível Superior-CAPES, Brazil (I.M. and B.N.). Zui Oporto wants to specially thank Dr. Juana Centellas Arias and the Instituto Oncológico Nacional-Caja Petrolera de Salud-IONCPS, Bolivia.

Open Access This article is distributed under the terms of the Creative Commons Attribution 4.0 International License (http://creativecomm ons.org/licenses/by/4.0/), which permits unrestricted use, distribution, and reproduction in any medium, provided you give appropriate credit to the original author(s) and the source, provide a link to the Creative Commons license, and indicate if changes were made.

Funded by SCOAP . $^{3}$

\section{Appendix: Conventions and notation}

\section{A.1 Conventions}

4D and 5D space-time indices: $\mu, \ldots=0, \ldots, 3$ and $\alpha, \ldots=0, \ldots, 4$.

3D and 4D space indices: $a, \ldots=1, \ldots, 3$ and $m, \ldots=$ $1, \ldots, 4$.

The de Sitter or anti-de Sitter groups $\mathrm{SO}(\mathrm{n}, \mathrm{N}-\mathrm{n})$ are collectively denoted by $(\mathrm{A}) \mathrm{dS}_{N}$. Their indices and corresponding invariant metrics are denoted by

(A) $\mathrm{dS}_{6}: \quad M, N, \ldots=0, \ldots, 5, \quad \eta_{M N}=\operatorname{diag}(-1,1,1,1,1, s)$,

(A) $\mathrm{dS}_{5}: \quad A, B, \ldots=0, \ldots, 4, \quad \eta_{A B}=\operatorname{diag}(-1,1,1,1, s)$,

where $s$ takes the values \pm 1 for $\mathrm{dS}$ or AdS, respectively. 4D Lorentz $\mathrm{SO}(1,3)$ indices are denoted by $I, \ldots=0, \ldots, 3)$, the corresponding metric being $\eta_{I J}=\operatorname{diag}(-1,1,1,1)$. These 
metrics and their inverses allow one to lower and raise the various group indices.

The respective Levi-Civita symbols are defined as

$$
\begin{aligned}
\varepsilon_{M N P Q R S} & =\left\{\begin{array}{l}
\varepsilon_{012345}:=1, \\
\varepsilon_{A B C D E 4}:=\varepsilon_{A B C D E},
\end{array}\right. \\
\varepsilon_{A B C D E} & =\left\{\begin{array}{l}
\varepsilon_{01234}:=1, \\
\varepsilon_{I J K L 4}:=\varepsilon_{I J K L},
\end{array}\right. \\
\varepsilon_{I J K L} & =\left\{\begin{array}{l}
\varepsilon_{0123}:=1, \\
\varepsilon_{0 i j k}:=\varepsilon_{i j k},
\end{array}\right.
\end{aligned}
$$

for the internal spaces, and

$$
\begin{aligned}
\varepsilon^{\alpha \beta \gamma \delta \varepsilon} & =\left\{\begin{array}{l}
\varepsilon^{01234}:=1, \\
\varepsilon^{\mu \nu \rho \sigma 4}:=\varepsilon^{\mu \nu \rho \sigma},
\end{array}\right. \\
\varepsilon^{\mu \nu \rho \sigma} & =\left\{\begin{array}{c}
\varepsilon^{0123}:=1, \\
\varepsilon^{0 a b c}:=\varepsilon^{a b c},
\end{array}\right.
\end{aligned}
$$

for the 5D and 4D space-times.

\section{A.2 Lie algebra basis}

A basis of the Lie algebra (a)ds $\mathrm{ds}_{6}$ of the group $(\mathrm{A}) \mathrm{dS}_{6}$ may be given by the 15 matrices $M_{P Q}=-M_{Q P}$ :

$$
\left(M_{P Q}\right)^{M}{ }_{N}:=-\left(\delta_{P}^{A} \eta_{N Q}-\eta_{P N} \delta_{Q}^{M}\right)
$$

satisfying the (a) $\mathrm{ds}_{6}$ commutation relations

$$
\begin{aligned}
{\left[M_{M N}, M_{P Q}\right]=} & -\eta_{M Q} M_{N P}-\eta_{N P} M_{M Q} \\
& +\eta_{M P} M_{N Q}+\eta_{N Q} M_{M P}
\end{aligned}
$$

One can decompose this basis according to representations of the 5D Lorentz group $\mathrm{SO}(1,4)$ as

$$
M_{M N}=\left\{\begin{array}{l}
M_{A B} \\
P_{A}:=\lambda M_{A 5}
\end{array}\right.
$$

where a positive dimensionful parameter $\lambda$ has been introduced, related to a cosmological constant $\Lambda \sim s \lambda^{2}\left(s=\eta_{55}\right)$ of a $5 \mathrm{D}$ gravitation theory. The commutation relations read now

$$
\begin{aligned}
{\left[M_{A B}, M_{C D}\right]=} & -\tilde{\eta}_{A D} M_{B C}-\tilde{\eta}_{B C} M_{A D}+\tilde{\eta}_{A C} M_{B D} \\
& +\tilde{\eta}_{B D} M_{A C}, \\
{\left[M_{A B}, P_{C}\right]=} & \tilde{\eta}_{A C} P_{B}-\tilde{\eta}_{B C} P_{A}, \\
{\left[P_{A}, P_{B}\right]=} & s \lambda^{2} M_{A B},
\end{aligned}
$$

with $\tilde{\eta}_{A B}=\operatorname{diag}(-1,1,1,1,1)$. The ten generators $M_{A B}$ generate the $5 \mathrm{D}$ Lorentz group, and together with the five generators $P_{A}$, generate the (A) $\mathrm{dS}_{6}$ group for $5 \mathrm{D}$ space-time. The $M_{A B}$ may be represented by the $5 \times 5$ matrices

$$
\left(M_{C D}\right)_{B}^{A}:=-\left(\delta_{C}^{A} \tilde{\eta}_{B D}-\tilde{\eta}_{C B} \delta_{D}^{A}\right) .
$$

The first line of A.4, namely

$$
\begin{aligned}
{\left[M_{A B}, M_{C D}\right]=} & -\eta_{A D} M_{B C}-\eta_{B C} M_{A D} \\
& +\eta_{A C} M_{B D}+\eta_{B D} M_{A C},
\end{aligned}
$$

but this time with the metric $\eta_{A B}=\operatorname{diag}(-1,1,1,1, s)$, gives the commutation rules of the Lie algebra of $(A) \mathrm{dS}_{5}$. Its decomposition according to representations of the 4D Lorentz group reads

$M_{A B}=\left\{\begin{array}{l}M_{I J} \\ P_{I}:=\lambda M_{I 4}\end{array}\right.$.

In the same way as above we have introduced the dimensionful parameter $\lambda$ related now to the cosmological constant of a 4D gravitation theory. Thus

$$
\begin{aligned}
{\left[M_{I J}, M_{K L}\right]=} & -\eta_{I L} M_{J K}-\eta_{J K} M_{I L} \\
& +\eta_{I K} M_{J L}+\eta_{J L} M_{I K}, \\
{\left[M_{I J}, P_{K}\right]=} & \eta_{I K} P_{J}-\eta_{J K} P_{I}, \\
{\left[P_{I}, P_{J}\right]=} & s \lambda^{2} M_{I J} .
\end{aligned}
$$

We are also interested in the full decomposition of the $(\mathrm{A}) \mathrm{dS}_{6}$ algebra according to representations of the Lorentz group $\mathrm{SO}(1,3)$ :

$$
\begin{aligned}
M_{I J}, \quad P_{I}:= & \lambda M_{I 5}, \quad Q_{I}:=\lambda M_{I 4}, \quad R:=M_{45}, \\
{\left[M_{I J}, M_{K L}\right]=} & -\eta_{I L} M_{J K}-\eta_{J K} M_{I L} \\
& +\eta_{I K} M_{J L}+\eta_{J L} M_{I K}, \\
{\left[M_{I J}, P_{K}\right]=} & \eta_{I K} P_{J}-\eta_{J K} P_{I}, \\
{\left[M_{I J}, Q_{K}\right]=} & \eta_{I K} Q_{J}-\eta_{J K} Q_{I}, \\
{\left[M_{I J}, R\right]=} & 0, \\
{\left[P_{I}, P_{J}\right]=} & s \lambda^{2} M_{I J}, \quad\left[Q_{I}, Q_{J}\right]=\lambda^{2} M_{I J}, \\
{\left[P_{I}, Q_{J}\right]=} & \lambda^{2} \eta_{I J} R, \\
{\left[P_{I}, R\right]=} & s Q_{I}, \quad\left[Q_{I}, R\right]=-P_{I} .
\end{aligned}
$$

\section{A.3 Dimensions}

The dimensions of the fields and the parameters of the theory, given in mass units, are

\begin{tabular}{c|c|c|c|c|c|c|c} 
& $\mathrm{d} s$ & $\omega^{I J}$ & $e^{I}$ & $\lambda$ & $\Lambda$ & $M_{I J}$ & $P_{I}$ \\
\hline $\operatorname{dim}$ & -1 & 1 & 0 & 1 & 2 & 0 & 1
\end{tabular}

\section{References}

1. C. Rovelli, Quantum Gravity. Cambridge Monographs on Mathematical Physics (Cambridge University Press, Cambridge, 2004)

2. T. Thiemann, Modern Canonical Quantum General Relativity. Cambridge Monographs on Mathematical Physics (Cambridge University Press, Cambridge, 2008)

3. C. Rovelli, F. Vidotto, Covariant Loop Quantum Gravity (Cambridge University Press, Cambridge, 2015) 
4. P.A.M. Dirac, Lectures on Quantum Mechanics (Dover Publications Inc., Mineola, New York, 2001)

5. M. Henneaux, C. Teitelboim, Quantization of Gauge Systems (Princeton University Press, Princeton, 1994)

6. E. Witten, (2+1)-Dimensional gravity as an exactly soluble system. Nucl. Phys. B 311, 46 (1988)

7. D. Lovelock, The Einstein tensor and its generalizations. J. Math. Phys. 12, 498 (1971)

8. C. Teitelboim, J. Zanelli, Dimensionally continued topological gravitation theory in Hamiltonian form. Class. Quantum Grav. 4, L125 (1987)

9. A.H. Chamseddine, Topological gauge theory of gravity in fivedimensions and all odd dimensions. Phys. Lett. B 233, 291 (1989). doi:10.1016/0370-2693(89)91312-9

10. M. Banados, C. Teitelboim, J. Zanelli, Lovelock-Born-Infeld theory of gravity. in J.J. Giambiagi Festschrift, ed. by H. Falomir, pp. 15-38 (World Scientific Publishing, Singapore, 1990)

11. M. Banados, C. Teitelboim, J. Zanelli, Dimensionally continued black holes. Phys. Rev. D 49, 975-986 (1994). doi:10.1103/ PhysRevD.49.975

12. J. Zanelli, Chern-Simons forms in gravitation theories. Class. Quantum Grav. 29, 133001 (2012). doi:10.1088/0264-9381/29/13/ 133001

13. A.H. Chamseddine, Topological gravity and supergravity in various dimensions. Nucl. Phys. B 346, 213 (1990)

14. J. Zanelli, Chern-Simons forms in gravitation theories. Class. Quantum Grav. 29, 133001 (2012)

15. A. Achucarro, P.K. Townsend, A Chern-Simons action for threedimensional anti-de sitter supergravity theories. Phys. Lett. B 180, 89 (1986)pagination
16. K. Isler, C.A. Trugenberger, A gauge theory of two-dimensional quantum gravity. Phys. Rev. Lett. 63, 834 (1989)

17. M. Bañados, L.J. Garay, M. Henneaux, The local degrees of freedom of higher dimensional pure Chern-Simons theories. Phys. Rev. D 53, 593 (1996). arXiv:hep-th/9605159

18. M. Bañados, L.J. Garay, M. Henneaux, The dynamical structure of higher dimensional Chern-Simons theory. Nucl. Phys. B 476, 611 (1996). arXiv:hep-th/9506187

19. I. Morales, B. Neves, Z. Oporto, O. Piguet (2016, work in progress)

20. J. Bernabeu, Gravitational waves in the presence of a cosmological constant. Phys. Rev. D 84, 063523 (2011). arXiv:1106.4511 [hepth]

21. J. Bernabeu, Gravitational waves in the presence of a cosmological constant (Erratum). Phys. Rev. D 86, 069904 (2012)

22. Planck 2015 Results. XIII. Cosmological Parameters. Planck Collaboration (P.A.R. Ade (Cardiff U.) et al.) (2015). arXiv:1502.01589 [astro-ph.CO]

23. A. Toloza, J. Zanelli, Cosmology with scalar-Euler form coupling. Class. Quantum Grav. 30, 135003 (2013). arXiv:1301.0821 [gr-qc]

24. T. Padmanabhan, Cosmological constant: the weight of the vacuum. Phys. Rep. 380, 235 (2003). arXiv:hep-th/0212290

25. Wolfram Research, Inc., Mathematica, Version 10.3, Champaign (2015) 